\title{
CRISIS Y FUTURO DE LA SEGURIDAD SOCIAL
}

\author{
Felipe Serrano Pérez \\ (Universidad del Pais Vasco / Euskal Herriko Unibertsitatea)
}

El objetivo que se persigue en este artículo es plantearse el futuro de nuestro sistema de seguridad social. Para ello, se procede al análisis de la crisis financiera que actualmente le amenaza, poniéndola en relación conun esquema general de interpretación de la crisis económica. Finalmente se pasa revista al proyecto, o proyectos parciales, que en estos momentos está implantando el gobierno con el objetivo de reformar el sistema.

\section{INTRODUCCION}

En un reciente seminario celebrado entre investigadotes europeos y latinoamericanos con el objeto de efectuar un estudio comparativo de nuestros respectivos sistemas de protección social se suscitó, ptácticamente des". de el inicio, el problema central que hoy tenemos planteado respecto al futuro de dichos sistemas: el de la teversibilidad o no de la participación del Estado en este campo de intervención.

El caso chileno, en donde recientemente se acaba de aprobar una ley privatizando el régimen de pensiones, era utilizado por los investigadores latinoamericanos como un ejemplo para advertir que, al menos en sus países, la solución definitiva a la crisis por la que atraviesan actualmente sus. sistemas de seguridad social podría pasar, si no por un total desmantela- 
miento de los sistemas públicos, sí al menos por la privatización de programas importantes, básicamente los programas de jubilación.

Los investigadores europeos nos limitábamos a constatar los efectos negativos que la crisis económica estaba originando en los niveles de protección social, pero sin atrevernos a avanzar ninguna hipotesis sobre el futuro que espera a nuestros sistemas de seguridad social.

Pero más allá del análisis comparativo de las distintas experiencias que fueron estudiadas, el problema de fondo que subyacía era el de intentar dar respuestas satisfactorias al problema más general que se había suscitado. A lo largo del seminario fuimos tomando conciencia de que, para avanzar en esta dirección, era necesatio profundizar en el análisis del origen de los sistemas de protección social; en la relación de estos sistemas con el mercado de trabajo; en cómo se había articulado la dinámica de la protección social con la dinámica de la acumulación del capital. En definitiva, era preciso estudiar a fondo, para cada uno de los países, de qué forma los sistemas de protección social, y de manera singular los sistemas de seguridad social, habían entrado en relación con el conjunto de variables socioeconómicas para acabar configurando un mapa de estabilidad económica que hoy se encuentra en crisis.

Una vez delimitados los campos de análisis, el problema del método se suscitó de manera inmediata. La crisis de los sistemas de seguridad social debía ser entendida como formando parte de esa crisis más amplia que experimentan las economías occidentales, de tal forma que el punto de artanque necesariamente debía pasar por una comprensión clara de la actual crisis de regulación del capitalismo.

En mi opinión, y en relación con este último aspecto señalado, el método de análisis nos debe permitir integrar una teoría de la crisis en una teoría que tenga como objetivo fundamental la explicación de la dinámica del capital a largo plazo, Io cual nos facultaria para entender las etapas de estabilidad y las etapas de crisis que caracterizan la historia del capitalismo como momentos distintos, y diferenciados entre sí, de esa dinámica. Es por esto por lo que el presente artículo se inicia esbozando estas líneas metodológicas fundamentales que nos permitan entender de una forma más clara, superando la mera descripción de acontecimientos, lo que está aconteciendo en la actual crisis económica con nuestro sistema de seguridad social. 


\section{UNA TEORIA DE LA CRISIS ECONOMICA}

El proceso de reproducción del capital descansa en la doble condición de extracción de la plusvalía y de tealización de la misma, de manera tal que se establezca una correspondencia entre la estructura de la producción y la estructura de las necesidades sociales. De forma sintética, las catacterísticas que definen cada uno de estos momentos del proceso del capital son señalados por Marx en los siguientes términos: «Las condiciones de explotación directa y las de su realización no son idénticas. Difieren no sólo en cuanto a tiempo y lugar, sino también conceptualmente. Unas sólo están limitadas por la fuerza productiva de la sociedad, mientras que las otras sólo lo están por la proporcionatidad entre las diversas ramas de la producción y por la capacidad de consumo de la sociedad. Pero esta capacidad no está determinada por la fuerza absoluta de producción ni por la capacidad absoluta de consumo, sino por la capacidad de consumo sobre la base de relaciones antagónicas de distribución. ${ }^{1}$

Los límites que actúan sobre las "condiciones de explotación directa» no son otros que aquellos que inciden directamente sobre el proceso de valoración del capital o, dicho en otros términos, los que encuadran el proceso de generación del plusvalor. Es al conflicto que enfrenta el capital con el trabajo al que nos tenemos que temitir para dar cuenta de aquéllos.

Entender estas condiciones que limitan la explotación directa a partir del conflicto significa que, implícitamente, estamos haciendo una determinada lectura de lo que Marx denomina en la cita reseñada «fuerza productiva de la sociedad». En efecto, frente a una lectura tecnológica de esta idea, que nos pondría en relación el proceso de valorización del capital con una teoría del desarrollo de la técnica, estamos implícitamente oponiendo la consideración de que las decisiones de innovación, de modificación de los procesos productivos, no son un dato exógeno que depende del grado de avance que en un momento histórico determinado haya alcanzado la investigación técnica. Por el contrario, la elección de técnicas por parte del capitalista está en relación directa con el mayor o menot grado de erosión que imponga la resistencia de la clase obrera, y con el mayor o menor grado de presión que ejerzan sus competidores en orden a valorizar un capital previamente avanzado.

Es particularmente conveniente insistir en este aspecto por la estrecha conexión que guarda con el análisis de la intervención estatal, y de forma singular con el análisis de la seguridad social. Una interpretación tecnolo-

1. Marx, K., El Capital, libro III, vol. 1, p. 313, Ed. Siglo XXI, 1981. 
gicista del proceso de valorización del capital vería en el Estado un mero agente desvalorizador del capital, estableciendo la relación entre la esfera privada y la esfera estatal en unos términos funcionales, de sobredeterminación de una sobre otra.

Con esto no estamos afirmando implícitamente que tesis como éstas sean falsas, sino, sencillamente, que no son suficientes 0 , mejor dicho, que tienen importantes sesgos interpretativos. La no consideración, o su inclusión de una forma secundaria, del conflicto, tanto entre el capital y el trabajo como entre las distintas fracciones del capital, impide aprehender que los procesos de etosión de las condiciones de explotación pueden adoptar formas que no son estrictamente económicas. El conflicto entre las partes enfrentadas se desarrolla en espacios distintos (el económico y el político), de donde emanan resultados contradictorios y potencialmente conflictivos para el capital que influyen en sus procesos de valorización, deteriorando o reforzando unas determinadas condiciones históricas de explotación de la fuetza de trabajo.

El proceso de definición de la actividad estatal es el resultado de unas prácticas en donde la relación de fuerzas entre las partes puede ser alterada en mayor o menor medida en función de la efectividad que demuestren en un momento determinado los mecanismos que filtran, en la esfera estatal, Jas distintas reivindicaciones de los agentes actuantes. Es necesario tener siempre en cuenta la posibilidad de comportamientos autónomos de lo po. lítico ${ }^{2}$ al hablar de condiciones de explotación. De igual forma que es preciso tener en cuenta que la propia definición de los mecanismos institucio. nales de integración del conflicto puede ser en un primer momento incluso contradictoria con las condiciones técnicas de acumulación con que el capital valoriza su dinero adelantado.

Permítasenos una breve digresión para tratar de consolidar las ideas que se acaban de exponer acerca del origen de la seguridad social. Un nexo teótico clásico en el pensamiento marxista pata establecer la relación entre fuerza de trabajo y gestión estatal de ésta, al menos por lo que a la actividad estatal que aquí nos ocupa se refiere, se estableció partiendo del «ejército incustrial de reserva». ${ }^{3}$ Esta tesis podría resumirse, aun a tiesgo de ser ex.

2. Cuando hablamos de acomportamiento autónomo de lo político" estamos pensando en una interpretación de la actividad estatal que se aleja de Ia idea de "autono. mía telativa» en tanto que principio de racionalidad en el ejercicio del poder político. Más bien estamos pensando en un concepto de «autonomía telativa» que revela las con. tradicciones consustanciales a la forma de dominación política que caracteriza el modo de producción capitalista, con una relación social que se recrea en dos espacios distintos (el económico y el político).

3. Véase, por ejemplo, el trabajo de Brunhoff, S. (de), Etat et Capital. Ed. Mas. péro, 1982. 
cesivamente simplista, en los siguientes términos: para el correcto funcionamiento del sistema económico capitalista es preciso que exista un «ejétcito industrial de reservam; ni el capital ni los trabajadores pueden hacerse cargo de su mantenimiento, ergo la intervención del Estado para imponer los «intereses de conjunto de la teproducción del capital» es inevitable.

En nuestra opinión, las tesis de este estilo soslayan el reto teórico que supone integrar en el análisis una concepción del Estado como un espacio de conflicto. Como un espacio por cuyos canales «los derechos burgueses democráticos», en los que Offe ve el elemento fundamental de contradicción de la forma de dominación política del capital, ${ }^{4}$ pueden penetrar las distintas reivindicaciones de los agentes actuantes y configurar, según su relación de fuetzas, el contenido definitivo de las funciones del Estado. La tesis de la seguridad social como conquista de la clase obrera, aunque presente en los trabajos del marxismo más funcionalista, solamente adquiere su verdadero significado cuando se acepta al Estado como un espacio en el que el conflicto acaba por determinar la configuración definitiva de sus funciones.

Concebir, entonces, los límites que actúan sobre las condiciones de ex* plotación directa a partir del conflicto no niega la presencia del cambio tecnológico, sino que, sencillamente, pone en relación a éste, o lo explica, a partir de la contradicción fundamental que se desarrolla en el modo de producción capitalista.

Por lo que se refiere al segundo monento del proceso del capital, el de la realización del beneficio, las propiedades de la mercancía —el ser valor de uso y valor de cambio a un mismo tiempo- obligan a afirmar, siguiendo a Marx, que uno puede explicarse absolutamente nada a partir de la relación entre la oferta y la demanda antes de estar desarrollada la base sobre la cual opera esta relación». 5

En efecto, si nos centramos en la formación de la oferta, sabemos que la producción de mercancías bajo el modo de producción capitalista no se organiza en función de las necesidades sociales, sino en función de la obtención de un plusvalor. «Nunca hay que olvidar que la producción de este plusvalor $-y$ la reconversión de una parte del mismo en capital, o sea, la acumulación, constituye una parte integrante de esa producción de plusvalor- es el objetivo directo y el motivo determinante de la producción capitalista.» ${ }^{\circ}$ Dicho con otras palabras, es el «valor de cambio» de la mercancía y no su «valor de uso" el que determina las actuaciones del capital.

4. Véase Offe, $\mathrm{C}$., «La abolición del control del mercado y el problema de la legi. timidad», en El Estado en el capitalismo contemporáneo. Edición a cargo de Sonntag, H. R., y Valecillas H., Ed. Siglo XXI, 1977.

5. Marx, K., El Capital, op. cit., pág. 230.

6. Ibíd., pág. 313 . 
Ahora bien, la oferta no solamente se caracteriza por estar organizada en función del plusvalor, sino que, además, es el resultado de una multitud de acciones individuales, de capitalistas individuales que actúan sin ningún tipo de conexión, bajo condiciones de producción $-\mathrm{y}$ por lo tanto de generación de beneficio- dispares, y conectadas únicamente por una misma dinámica, o por un mismo denominador común: la obtención del máximo beneficio. Este hecho nos remite a la proporcionalidad entre las diferentes ramas de la producción como la primera limitación que apuntaba Marx en el proceso de realización de beneficio.

Las necesidades sociales, por su parte, deben ser analizadas a partir del concepto de «valor de uso» de las mercancías. Desde esta perspectiva, la demanda se puede considerar como un elemento exógeno al capital. La existencia de unas necesidades sociales, independientemente de que exista o no una producción que las cubra, es algo que, en principio, podríamos pensar que escapa a la voluntad y al control del capital. Sin embargo, no puede decirse lo mismo de su volumen, ya que, como señala Marx, «las necesida* des sociales, es decir, aquello que regula el principio de la demanda, están condicionadas en lo fundamental por la relación tecíproca entre las diversas clases y por su respectiva posición económica, esto es, principalmente y en primer lugar por la proporción entre el plusvalor global y salario, y en segundo lugar por las diversas partes en las cuales se escinde el plusvalor (ganancia, interés, tenta de la tietra, impuestos». ${ }^{7}$ Dicho en otros términos, los límites que afectan el momento de realización de la plusvalía, desde el lado de la demanda, son unos límites directamente emanados de la propia lógica del proceso de reproducción del capital.

Si éstos son los límites que actúan sobre la esfera de la realización, el problema de ajuste entre las necesidades sociales y la oferta se plantea en términos de indeterminación, de problema a resolver: «No hay una conexínn necesaria, sino solamente casual, entre la cantidad global de trabajo social aplicada a un artículo social, es decir, entre la parte alícuota de su fuerza de trabajo global que emplea la sociedad para la producción de ese artículo, o sea, entre el volumen que ocupa la producción global, por una parte, y el volumen en que la sociedad reclama la satisfacción de las necesidades sociales por ese artículo determinado, por la otra.» ${ }^{8}$ Esta divergencia emana, in. sistimos, de la duplicidad de propiedades que permite que la mercancía sea un valor de cambio y un valor de uso.

La solución a este problema, o, en otras palabras, el proceso a través del cual el sistema es capaz de ajustar la producción al volumen de necesi-

7. Ibíd., pág. 230.

8. Ibíd., pág. 236. 
dades sociales, necesariamente se ha de arrancar de la causa fundamental que propicia la producción de tipo capitalista: la obtención del beneficio. Es la competencia entre capitales, la emigzación de los capitales de unos sectores de la producción a otros en busca del máximo beneficio, lo que, al ir igualando las condiciones de ptoducción entre los diferentes capitales, provoca las necesarias modificaciones en la configuración de la oferta de manera que ésta se adapte a la estructura de las necesidades sociales.

En línea con lo apuntado, el GRREC ${ }^{9}$ ha acertado a definir la dinámica a largo plazo del capitalismo a partir de la idea de «regulación», entendida ésta a su vez como la articulación, en un espacio deternoinado (concepto de «sistema productivo»), de las dos leyes del beneficio capitalista (la «ley de la baja tendencial de la tasa de ganancia» y la «ley de la igualación de las tasas de ganancia») de acuerdo con unas normas o «procedimientos sociales de regulación» (funciones del Estado).

Estos tres conceptos: «sistema productivo», «leyes del beneficio»y "procedimientos sociales de regulación», permiten periodizar la dinámica histórica del capital, mostrando la alternancia de etapas de estabilidad estructural del sistema con etapas de crisis; etapas estas últimas en las que se asiste al derrumbamiento de las bases de regulación de los períodos de estabilidad previos, es decir, al fin de una determinada manifestación de «coherencia» entre esas tres categotías apuntadas y al establecimiento de nuevos procedimientos, o de un nuevo «modo de regulación», de articulación «coherente» de las dos leyes del beneficio en un espacio determinado con nuevas normas o nuevos procedimientos sociales de regulación.

Apuntemos, para evitar posibles malentendidos, que las crisis económicas no son algo necesario para el proceso de reproducción del capital, en la medida en que históticamente podemos concluir de ellas que han actuado como revulsivo de aquél. Son, por el contrario, una consecuencia lógica del propio proceso del capital cuya superación por medio de la creación de nuevas relaciones, o de un nuevo modo de regulación, es algo imprevisible en tanto que no depende de la mente de un planificador, sino de la interacción de intereses contrapuestos y contradictorios sobre los que, a priori, es factible pensar sobre su imposibilidad de compatibilidad o de «coherencia».

De lo que se acaba de indicar se desprende una idea que cobra vital importancia para el asunto que nos ocupa. Las crisis se presentan como el momento histórico en el cual se asiste al desmantelamiento de todo el entramado de relaciones entre las variables socio-económicas que habían caracterizado la anterior etapa de estabilidad. Y, de forma simultánea, la crisis se presenta también como un momento de construcción de algo nuevo, como un

9. GRREC, Crises et Regulation. Recueil de textes. 1979-1983, Ed. PUG, 1983. 
momento de recomposicion de nuevas relaciones entre las variables que pueden conducir al capitalismo hacia una nueva etapa de estabilidad.

Esta caracterización de la crisis como un momento de transición, dinamizada por el conflicto que enfrenta a los distintos agentes actuantes, introduce una indeterminación teórica en cuanto a la posibilidad de prever la configuración definitiva, si es que se alcanza, de una nueva etapa de estabilidad, y, consiguientemente, respecto a la posibilidad de prever lo que puede ocurrir con los sistemas de previsión social. Podremos tener una idea más o menos clara de cómo la crisis está erosionando el sistema de seguridad social, pero resulta difícil responder a la cuestión central que avanzamos al inicio de estas páginas sobre la reversibilidad o irreversibilidad de dicho sistema.

\section{LA CRISIS EN SU CONFIGURACION ACTUAL: SU IMPACTO SOBRE NUESTRO SISTEMA DE SEGURIDAD SOCIAL}

Más allá de la explicación que dernos sobre el surgimiento de los sistemas de seguridad social, nunca debemos olvidar que su desarrollo ha sido facilitado en la medida en que aquéllos se han podido imbricar con la dinámica económica. Es cierto, y el análisis histórico lo demuestra, que han existido fricciones y contradicciones entre la dinámica de la política social y la dinámica económica. Sin embargo, hasta el desencadenamiento de la actual crisis económica, se había logrado superar estas contradicciones sin cuestionar los límites que enmatcaban el proceso de acumulación. La política social y la política económica se presentaban como dos prácticas sociales de regulación compatibles entre sí.

Podríamos profundizar más y mostrar qué tipo de compatibilidad se estableció en los distintos países atendiendo a las particulares circunstancias que caractetizaton su desartollo económico. Mostras cómo, al menos para el caso español, la articulación entre la dinámica económica y la política social no alcanzó grados de compatibilidad tan intensos como en la mayoría de los países europeos, sino que, por el contrario, el desartollo de la política social quedó profundamente lastrado por las necesidades del proceso de acumulación. ${ }^{10}$ Sin embargo, para el objetivo que aquí se persigue, estudiar la relación existente entre la crisis económica y la crisis de la seguridad social, puede considerarse secundario insistir en el desarrollo expositivo de

10. Serrano Pérez, F., "Crisis econónica y crisis de la seguridad social», en Información Comercial Española, núm. 665, 1989. 
esta relación. Aunque conviene señalar que la crisis diferencial que marca nuestro sistema de seguridad social, con unos niveles de prestación sustancialmente más bajos que los gatantizados en la Comunidad, encuentra su raíz en la tardanza con que se diseña la nueva política social a principios de la década pasada.

Es preciso aclarar de forma previa, aunque sea reiterar algo sobradamente conocido, que los sistemas de seguridad social de base contributiva se presentan como un conjunto de programas públicos, sostenidos por la contribución obligatotia de trabajadores y empresarios y transferencias estatales, a través de los cuales los trabajadores-contribuyentes adquieren el derecho a percibir un conjunto de servicios cuando ciertos acontecimientos interrumpen su capacidad de trabajar (vejez, invalidez, enfermedad, etc.). Este dato sobre las fuentes de financiación es particularmente importante para poder entender los márgenes dentro de los cuales cabe hablar de una crisis financiera del sistema de seguridad social cuando se alteran las condiciones económicas que permiten el flujo continuado y sostenido de la renta necesaria para contribuir al pago de dichas prestaciones.

Hecha esta aclaración, tal vez la mejor manera de presentar la relación entre la crisis económica y la crisis de la seguridad social sea planteando que, en la situación actual, se ha roto la compatibilidad entre política social y política económica que, como se apuntaba anteriormente, había carac. terizado la etapa de estabilidad económica de las décadas pasadas. La ruptura de esta compatibilidad hay que buscarla, al menos en nuestra opinión, en la incapacidad de las contratendencias que garantizan una expansión «estable» de la acumulación de capital para superar las tendencias a la crisis económica que están siempre presentes en la dinámica del capital (ruptura de la articulación de las dos leyes del beneficio).

Dicho en otras palabras: la caida de la tasa de ganancia ha obligado a redefinir los objetivos sociales y económicos, y, en consecuencia, a instrumentar políticas económicas de gestión de la crisis que entran en franca contradicción con la consecución de los objetivos sociales sobre los que se había fundado el consenso social en la antetior etapa de estabilidad económica. Son las políticas de tecuperación del excedente empresatial las que están erosionando los niveles de prestación que ofrece la seguridad social. $Y$, contradicción fundamental de estas estructuras de protección social, justamente en el momento en que, como consecuencia de la crisis, existe una mayor demanda sobre estos programas sociales.

Pero, ¿cómo se establece esta relación contradictoria entre la política social y la política económica? A finales de los años setenta se asiste a un giro en las políticas de gestión de la crisis que tenía por objetivo restablecer lo que podríamos denominar un encadenamiento macroeconómico «clá- 
sico». Es decir, la recuperación económica depende de la inversión y ésta, a su vez, depende directamente de la obtención de beneficios por parte del empresariado. La acción estatal, por tanto, debía centrarse en la eliminación de aquellas barteras que impedían una rápida recuperación del excedente. Una vez que este objetivo se hubiese conseguido, el mercado se encargaría de realizar lo demás. Es cierto, y sería demagógico olvidarlo, que la acción del Estado también debía dirigitse a facilitar que la recuperación de la inversión se hiciese sobre nuevas bases tecnológicas, lo que añadía una carga suplementaria a la actividad estatal, obligándola a diseñar planes de actuación sectoriales complementarios con las políticas de recuperación del beneficio.

Desde una perspectiva teórica la recuperación del excedente empresarial puede abordarse de distintas formas. En primer lugar, puede considerarse como un dato el coste del factor trabajo, de tal forma que la tecuperación de la productividad necesariamente ha de abordarse a través de la innovación técnica. En segundo lugar, la recuperación de la productividad, como paso imprescindible para recuperar el beneficio, puede lograrse actuando sobre el coste del factor trabajo, bien sea reduciendo las tasas de crecimiento vía contenciones salariales, bien reduciendo plantillas, o mediante una combinación de ambas ptácticas. Y, en tercer lugar, la recuperación del excedente puede obtenerse por una combinación de las dos políticas mencionadas.

En España, hacia finales de los años setenta, se opta por la política de disciplinar el crecimiento del coste del factor trabajo. Las reducciones de plantillas, la flexibilización del mercado de trabajo y la contención del crecimiento de los costes salariales han sido las políticas centrales que han permitido la recuperación del beneficio empresarial.

Los Pactos de la Moncloa apazecen como punto de referencia central. Su objetivo fundamental efa la contención de la espiral inflacionista, disciplinando el crecimiento de los agregados monetatios y de los costes laborales. Estos eran frenados mediante la alteración en el procedimiento de negociación de los incrementos salariales y con la reducción de las cuotas empresariales a la Seguridad Social.

La reducción de las aportaciones empresariales al sistema de seguridad social se planteaba como una medida que debía ser compensada por un incremento de las aportaciones estatales. Aportaciones que se presentaban como compensadoras de los potenciales desequilibrios financieros en que incurriría el sistema ante la reducción de cuotas. Igualmente se apuntaba que el Estado debía hacerse cargo progresivamente de la financiación del seguro de desempleo. En conjunto, por tanto, se contemplaba una modificación de la estructura financiera tendente a descargar costes a las empresas incrementando la participación estatal. 
Bloqueada la posibilidad de financiar el incremento del gasto en protección social tecurriendo a un incremento de las cotizaciones, la solución a los: déficit fnancieros en que podía incurrir la Seguridad Social se desplazaban hacia el Estado. Esta solución, sin embargo, pronto quedatía también bloqueada. En efecto, una política monetaria testrictiva tarde o temprano acaba por obligar a disciplinar el ritmo de expansión del gasto público, y esto, unido a un incremento de responsabilidades en materia de política industrial, necesariamente había de desembocar en la fijación de un límite máximo de transferencias del Estado a la Seguridad Social.

Paralelamente a esta política de fijación de topes financieros, se ha venido racionalizando la gestión del sistema de seguridad, iniciado con el RDL $36 / 1978$, por el que se consigue la unificación de todos los recursos financieros del sistema, y continuado por todo un conjunto de teformas parciales entre las que cabe destacar los intentos por corregir los desequilibrios financieros entre los distintos regímenes. Sin embargo, todo este conjunto de medidas ha sido incapaz de aumentar el margen de maniobra para que el sistema pudiese dar respuesta a las continuas demandas sociales que apa-. recen como resultado de la crisis. La restricción financiera es fuerte.

El resultado final no ha sido otro que una profunda erosión de los niveles de protección, que ya eran sustancialmente más baj̧os que los de la media comunitaria. El porcentaje de participación de los gastos de seguridad social en relacion al PIB permanece estancado entre el 12 y el 12,5 por $100^{\circ}$ desde 1981. La pensión media que garantiza el sistema de seguridad social sigue siendo inferior al salario mínimo intetprofesional, sin contar con la teducción que se ba oxiginado en las zuevas pensiones de jubilación por efecto de la ley $26 / 85$, conocida popularmente como la «ley de pensiones». La tasa de cobertura del seguro de desempleo se sitúa por debajo del 35 por100. El gasto sanitatio se ha estancado, mientras que la población protegida: ha ido aumentando, lo que arroja como resultado una disminución del gasto. sanitario por persona.

\section{IV. ¿TIENE FUTURO LA SEGURIDAD SOCIAL?}

Volvamos al problema central con el que iniciábamos estas páginas: el de la reversibilidad o no de la participación del Estado en el campo de la protección social y, de forma singular, en el de la Seguridad Social. Coherentes con nuestro método de análisis nos hemos limitado hasta el momento a mostrar cómo se está desmontando todo un entramado social que 
marcó intensamente la anterior etapa de estabilidad económica. Las políticas de gestión de la crisis lo están erosionando.

La crisis, sin embargo, como también se señaló anteriormente, no es sólo el momento histórico en el que se produce el desmantelamiento de to existente, sino también el momento histórico en el que se asiste a la construcción de algo nuevo. No obstante, la redefinición de una nueva frontera entre lo público y lo privado to es un proceso fácil de predecir. Plantear el futuro de nuestro sistema de seguridad social implica introducir en el análisis elementos que trascienden el campo estrictamente económico y que forman parte de la sociología política.

En efecto, el debate actual sobre la reforma de la Seguridad Social no es un debate estrictamente técnico; es decir, no gira exclusivamente en torno a la búsqueda de un nuevo modelo financiero que permita cubrir la expansión del gasto social que se deriva de la situación actual de crisis, así como del proceso de envejecimiento de la población. Junto a este problema «técnico», que no puede ser pensado olvidando la política económica, se encuentran elementos de discusión que muestran los planteamientos ideológicos de los distintos agentes actuantes.

La crítica frontal que el pensamiento más conservador ha dirigido a la participación del Estado en la economía se encuentra en la base de algunos de los proyectos de reforma del sistema de seguridad social defendidos, por ejemplo, por el sector empresarial español. Recuérdese los términos en que la CEOE formuló en la Comisión del ANE, que se creó para la reforma de la Seguridad Social, un sistema basado en tres niveles."

Con esta afirmación no se pretende descalificat de un plumazo la solución de un sistema de seguridad social en tres niveles: un primer nivel asistencial financiado con cargo a las transferencias del Estado; un segundo nivel contributivo y obligatorio, y un tercer nivel libre y complementario. La asistencia sanitaria cortería también con cargo a los ingresos generales del Estado. El problema aparece cuando se trata de dar contenido a los distintos niveles $y$, fundamentalmente, al nivel obligatorio $y$ al nivel complementario.

En efecto, permitir legalmente que la población pueda acceder a cubrir el riesgo de jubilación mediante la suscripción de un seguro voluntario en el mercado no es algo criticable. Sí lo puede ser, sin embargo, dado el estado actual en que se encuentra nuestro sistema de seguridad social, el que este recurso esté bonificado fiscalmente. Ahora bien, para que no se establezca una relación «perversa» entre el nivel obligatorio y el nivel libre, deben to-

11. Serrano Pérez, F., «Las destegulaciones en la crisis: el caso de la seguridad social española», en Economies et Sociétés, núm. 31, 1989. 
marse las suficientes garantías para que la pensión que devengue el nivel obligatorio sea suficiente, de tal forma que no se obligue a la población a recurrir al nivel complementario si desea tener una pensión digna.

$\mathrm{El}$ problema de la financiación de ese segundo nivel se convierte enton. ces en el núcleo central de la reforma. Desde diversos ámbitos se han sugerido distintas alternativas financieras para garantizar los recursos necesarios. Así, se ha propuesto que el nivel contributivo se fnanciase mediante una reducción de cuotas compensada por las aportaciones de un aumento sobre el Impuesto del Valor Añadido. Se ha propuesto, igualmente, que los recursos necesarios se obtengan mediante un incremento de las aportaciones estatales. Otra de las medidas barajadas, aunque no con gran insistencia en nuestro país, consiste en desplazar al conjunto del empresariado, mediante un impuesto especial sobre los beneficios, una parte del coste total, con una modificación correctora en la estructura de cotizaciones.

En España, y juzgando por las últimas medidas que están siendo adop* tadas por el Gobierno, puede afirmarse que la reforma del sistema camina por la ditección que estamos apuntando. La contingentación que se ha efectuado de las transferencias estatales, asignándolas por capítulos de prestaciones en el último ejercicio económico, unido a la reforma del régimen de pensiones desarrollado en el verano de 1985 y la que está en curso en ma* teria de pensiones asistenciales, permite afrmar que el proyecto de reforma de la Seguridad Social ya está en matcha.

La estructura de financiación por la que parece haber optado el Gobierno para garantizar los recursos necesarios que han de cubrir el segundo nivel sigue basárdose en las cotizaciones obligatorias, y se refuerza la relación existente entre lo cotizado y lo percibido en forma de pensión, aunque levemente matizada esta relación por la inclusión de unos niveles mínimos de pensión.

¿Cuál va a set la traducción, por lo que a intensidad de la prestación se refiere, de este proyecto de reforma? Poco se puede decir en este momento, aunque los análisis que se han venido desarrollando con posterioridad a la entrada en vigor de la ley del 85 han permitido detectar una caída, relativamente importante, de la pensión media percibida por los nuevos jubilados (en el período 1985-1987 las nuevas pensiones se redujeron altededor de un 8 por 100).

Ahora bien, más allá del modo en que se acaben por traducir estas reformas parciales en los riveles de protección, conviene destacar que este proyecto de reforma (nos referimos a las pensiones públicas) solamente tendrá una cierta viabilidad si se consigue desligar de la marcha económica, de la política económica para ser más exactos, la política de cotizaciones. Hemos tenido ocasión de mostrar anteriormente de qué manera formaban parte las 
cotizaciones empresariales de un conjunto de políticas más amplias tendentes a tecuperar el bebeficio empresarial. Pues bien, o se consigue romper esta ligazón, y entonces se pasa a calcular las cotizaciones en función de las necesidades que se tengan de recursos para cubrir el monto anual que suponga el pago de las pensiones, o la teforma no nos sacatá de la situación en la que nos encontrataos.

Esta política de reforma que parece detectarse del conjunto de actuaciones que viene llevando a cabo el Gobierno significa un cambio importante en la filosofía sobre la que se habían venido basando los sistemas de seguridad social. En efecto, el principio de solidaridad que, digámosio también, no es sinónimo de explotación de unos grupos laborales sobre otros, como ha venido ocurriendo en nuestro sistema, ha sido sacrificado en beneficio de la equidad individual: la pensión de jubilación debe estar en relación directa con la cotización del individuo.

En el momento en el cual nos hallamos resulta, sin embargo, difícil pronunciarse de forma tajante acerca del futuro definitivo que le espera a nuestro sistema de seguridad social. La fuerte contestación social que está tecibiendo la política económica del Gobierno también afecta las medidas de reforma del sistema que hemos venido comentando. El que estas medidas se consoliden depende en gran parte de la aceptación que reciban por parte del conjunto de la sociedad. No en vano todas las recomendaciones efectuadas tanto por la OIT como por la Comunidad Europea insisten en la necesidad de pactar y consensuar la reforma de los sistemas de seguridad social como única forma de garantizar la viabilidad a los planes que se diseñan para su conservación.

Pero tampoco hay que olvidar que no todas las posturas sobre la reforma del sistema inciden en el mantenimiento del carácter público de éste. Las voces que reciaman un desentendimiento del Estado en este campo y una potenciación del metcado en el suministro de soluciones para los riesgos contemplados por el sistema son importantes.

Esta confrontación de fuerzas de signo contrario hace difícil saber cuál será la resultante final. Es cierto que el sistema de seguridad social está fuertemente arraigado en la sociedad, lo que dificulta grandemente una actuación radical del poder político tendente a su desmantelamiento. Pero también es cietto que, tal como parece presentarse la reforma del Gobierno, es de esperar que los niveles de prestación se reduzcan aún más, dando un mayor protagonismo al mercado. Algunas personas, entre las que me en. cuentro, son de la opinión que una mayor teducción de los niveles de prestación equivale a un desmantelamiento de facto del sistema de seguridad social. 